\title{
Calibrating corneal material model parameters using only inflation data: An ill-posed problem
}

\author{
S. Kok ${ }^{1, *, \dagger}$, N. Botha ${ }^{1,2}$ and H. M. Inglis ${ }^{1}$ \\ ${ }^{1}$ Department of Mechanical and Aeronautical Engineering, University of Pretoria, Pretoria, 0002, South Africa \\ ${ }^{2}$ Advanced Mathematical Modelling, CSIR Modelling and Digital Science, PO Box 395, Pretoria, O001, South Africa
}

\begin{abstract}
SUMMARY
Goldmann applanation tonometry (GAT) is a method used to estimate the intraocular pressure by measuring the indentation resistance of the cornea. A popular approach to investigate the sensitivity of GAT results to material and geometry variations is to perform numerical modelling using the finite element method, for which a calibrated material model is required. These material models are typically calibrated using experimental inflation data by solving an inverse problem. In the inverse problem, the underlying material constitutive behaviour is inferred from the measured macroscopic response (chamber pressure versus apical displacement). In this study, a biomechanically motivated elastic fibre-reinforced corneal material model is chosen. The inverse problem of calibrating the corneal material model parameters using only experimental inflation data is demonstrated to be ill-posed, with small variations in the experimental data leading to large differences in the calibrated model parameters. This can result in different groups of researchers, calibrating their material model with the same inflation test data, drawing vastly different conclusions about the effect of material parameters on GAT results. It is further demonstrated that multiple loading scenarios, such as inflation as well as bending, would be required to reliably calibrate such a corneal material model.
\end{abstract}

KEY WORDS: inverse problem; ill-posed; material coefficient calibration; elastic fibre-reinforced constitutive model; inflation test; Goldmann applanation tonometry; cornea

\section{INTRODUCTION}

Corneal biomechanical properties are crucial to understanding the response of the cornea to various surgical procedures (e.g. refractive surgery [1]), as well as to accurately measure other properties such as intraocular pressure (IOP) [2, 3], an important risk identifier for glaucoma. IOP is quantified using tonometry of which Goldmann applanation tonometry (GAT) is the 'gold standard' against which all other tonometers are calibrated [4]. During tonometry, the indentation resistance of the cornea is measured by applying a force until an applanated area of $7.35 \mathrm{~mm}^{2}$ is obtained. IOP is then inferred from the measured force using the Imbert-Fick law [5]. IOP measurement is known to be sensitive to corneal properties [2,3], in contradiction of the initial design premise of GAT [5].

The corneal properties that could potentially influence GAT include the cornea geometry (thickness, diameter and curvatures), as well as the corneal material properties. Geometrical changes can be investigated easily in numerical models, because there is no ambiguity in interpretation. The effect of uncertainty in material properties is, however, more difficult to investigate, as the material parameter variation cannot be isolated from the choice of a material model. For every material model chosen, the material model parameters must be calibrated to some experimental data. Only once

\footnotetext{
*Correspondence to: S. Kok, Department of Mechanical and Aeronautical Engineering, University of Pretoria, Pretoria, 0002 , South Africa.

†E-mail: schalk.kok@up.ac.za
} 
calibrated can the material parameter values be varied to quantify the effect of material property uncertainty on GAT results. This approach has two inherent dangers: first, an unreasonable material model might be selected; second, even for a reasonable choice, the material model parameters found during calibration might be unreliable.

The material model calibration process, also known as identification of the corneal material coefficients, is an inverse problem as these parameters are not known and should be derived from experimental data. An inverse problem is often ill-posed, defined as a problem having no unique solution (i.e. it has multiple solutions) or for which the solution is unstable (i.e. small variations in the experimental data could lead to large variations in the solutions) [6]. The unreliable predictions referred to earlier are a direct consequence of the ill-posed nature of the model calibration process.

In the case when an inappropriate material model is chosen, the effect of varying a specific material parameter in this material model could then produce misleading results. There should, however, be early indications of this error, because it is unlikely that an incorrect material model can accurately match the experimental data. Of more concern is the case where a reasonable material model is chosen, but unreliable material parameters are still obtained, and as a consequence, when applied to a different loading scenario, unreliable predictions result.

It has been shown by Studer et al. [7] that several studies utilising similar material models (an elastic fibre-reinforced model using either a neo-Hookean or Mooney-Rivlin law for the isotropic definition) obtained significant differences in material coefficients. These material coefficients were calibrated using an inverse finite element (FE) approach to exclusively fit experimental inflation test data. Studer et al. [7] proceeded to generate two sets of artificial data, consisting of only a single curve for each set, by considering the deformation of a plate along the fibre orientation as well as perpendicular to the fibre orientation. They employed a similar material model definition as the elastic fibre-reinforced model. They concluded that when calibrating a corneal material model, or any other soft biological tissue, it is necessary to use as many distinct experimental data sets as are available. However, it was not quantified what the effect of the material model choice or what the effect of the variation in material coefficients is on applications of corneal modelling such as tonometry or surgical procedures.

In this paper, we select a biomechanically motivated anisotropic fibre-reinforced hyperelastic material model for the cornea. We calibrate the material model parameters naively, by considering only the single experimental loading case of inflation testing. We demonstrate that we can fit each curve in the experimental data set closely. In some cases, a single curve from the data set can be fit using dramatically different material parameter values, a hallmark of an ill-posed problem. If these different material parameter values are used in the subsequent simulation of GAT, which incorporates both inflation and bending loading, the predicted IOP will vary significantly. In addition, the numerically predicted sensitivity of GAT to material parameter variation is then found to be unreliable. This behaviour is explained through a simple model of tension followed by bending, applied to a thin strip of the corneal material.

\section{FINITE ELEMENT MODEL OF THE CORNEA}

The FE model development is detailed in this section. The experimental data set used in the study is discussed first, in order to guide the development of the FE model. Next, the geometric description of the cornea is discussed, followed by the selected constitutive model. Descriptions of the numerical inflation test and the numerical GAT test conclude this section.

\subsection{Experimental data set}

Corneal material properties are primarily quantified by means of two ex vivo testing methods, namely inflation testing and strip extensometry. During an inflation test, a whole corneal specimen is clamped onto a pressure chamber and then inflated beyond its physiological limitations. In a strip extensometry test, a corneal strip of constant width, excised from a whole cornea, is clamped into a slow-rate tension machine and pulled in tension. The strip extensometry test is used less widely 


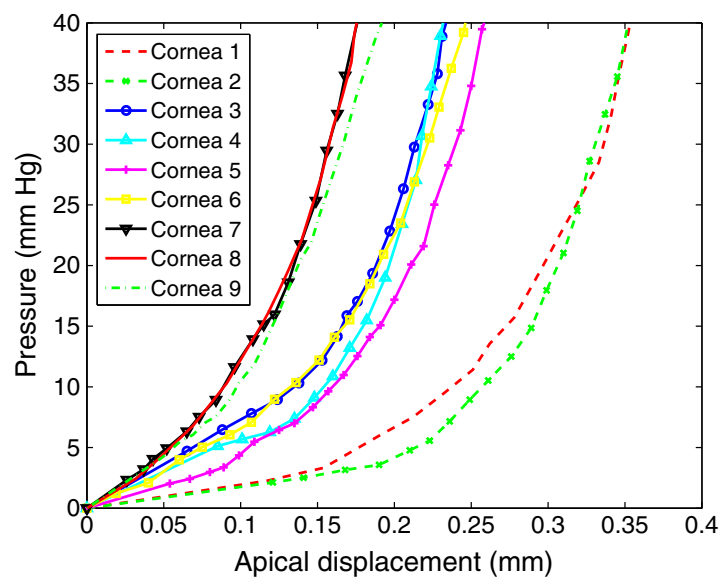

Figure 1. Experimental inflation test data results from Elsheikh et al. [12].

than the inflation test because it is believed to be less reliable $[8,9]$. As a result, numerical investigations often use only experimental inflation test data results to calibrate the corneal material models. However, there are a few studies that have used experimental data from both the inflation and strip extensometry tests with which to calibrate corneal material models to obtain a unique set of material coefficients [10,11].

Two sets of experimental inflation test data available in the literature, those of Bryant and McDonnell [1] and Elsheikh et al. [12], have been used to calibrate corneal material models. The data set of Elsheikh et al. is used in this study, because the tests were performed at $37^{\circ} \mathrm{C}$ and the geometric variation of the corneas is described statistically. In contrast, the Bryant and McDonnell tests were performed at $21{ }^{\circ} \mathrm{C}$, the epithelium was removed mechanically, and no information is provided about the geometric variation of the corneas. The inflation data for the Elsheikh data set are depicted in Figure 1, where it is evident that there is a wide dispersion in the experimental results. Most numerical studies only consider a single curve from such a dispersed data set. However, in this study, all the available curves from the experimental inflation test data will be considered.

\subsection{Geometric model of the cornea}

A number of possibilities exist to describe the cornea geometry. These range from simple analytical expressions (spherical, conicoid etc.) to detailed descriptions of the surface topography $[13,14]$. However, because of the limited number of geometric variables reported in the experimental study by Elsheikh et al., only the simple analytical expressions can be utilized in this study.

Carney et al. [15] discussed several mathematical models that have been used to describe the aspherical shape of the cornea. The simplest of these is a rotationally symmetric conicoid. The axis of rotation of the cornea is the optical axis (Z-axis), leading to the general equation for a 3D conicoid with the origin at $\left(X_{o}, Y_{o}, Z_{o}\right)$ on the anterior surface [15]:

$$
\left(X-X_{o}\right)^{2}+\left(Y-Y_{o}\right)^{2}+(1+Q)\left(Z-Z_{o}\right)^{2}-2 R\left(Z-Z_{o}\right)=0,
$$

where $R$ is the radius of curvature at the apex, $Q$ is the surface asphericity parameter, $X$ is the distance along the equator axis and $Y$ is the distance along the sagittal axis. The asphericity parameter, $Q$, defines the type of conicoidal surface where $Q>0$ refers to an oblate ellipsoidal surface, $Q=0$ to a spherical surface, $-1<Q<0$ to a prolate ellipsoidal surface, $Q=-1$ to a paraboloidal surface and $Q<-1$ to a hyperboloidal surface. The experimental data set by Elsheikh et al. [12] only reports four geometric variables, namely the central corneal thickness (CCT), the peripheral corneal thickness (PCT), the apical rise $(H)$ and the average limbal diameter $\left(2 R_{i}\right)$. For each of these variables, the mean and standard deviation are reported, as summarized in Table I. Elsheikh et al. use the spherical surface model $(Q=0)$ to describe both the anterior and posterior surfaces of the cornea. Although literature $[3,16]$ recommends asphericity parameter values of $Q_{a}=-0.18$ and 
Table I. Mean \pm standard deviation for the four geometric variables reported by Elsheikh et al. [12].
$\mathrm{CCT}(\mathrm{mm})$
PCT (mm)
$H(\mathrm{~mm})$
$2 R_{i}(\mathrm{~mm})$
$0.572 \pm 0.058$
$0.779 \pm 0.042$
$2.324 \pm 0.229$
$11.252 \pm 1.148$

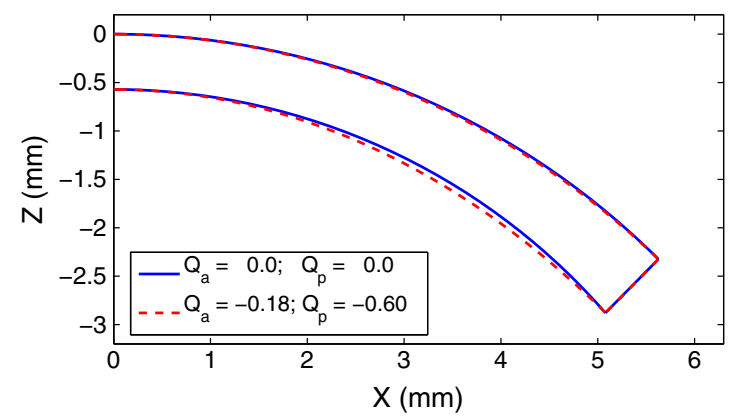

Figure 2. Effect of the asphericity parameter $Q$ on the cornea geometry.

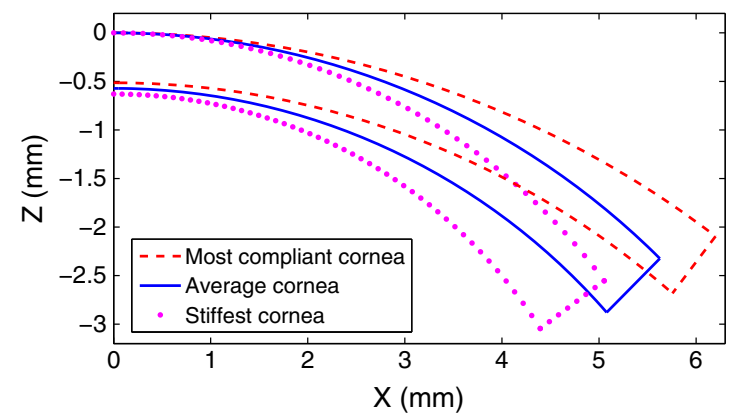

Figure 3. Effect of the variation in the four geometric properties on the final cornea geometry.

$Q_{p}=-0.60$ for the anterior and posterior surfaces, respectively (for pressurized corneas), Figure 2 demonstrates that the differences compared with using $Q=0$ are marginal. In this figure, the mean values for all the geometric variables were used.

To demonstrate the geometric variability due to the reported variation within the sample, three specific cornea geometries are generated. The first geometry is generated using the mean values in Table I. The other two geometries are generated by perturbing each of the mean values of the four geometric variables by adding or subtracting one standard deviation. The most compliant cornea geometry will result when subtracting one standard deviation from CCT, PCT and $H$, while adding one standard deviation to $2 R_{i}$. This results in a thin, large, flat cornea. The stiffest cornea geometry will result when the signs are changed on these perturbations. This in turn results in a thick, small, steep cornea. These three geometries are depicted in Figure 3. Comparing Figures 2 and 3, it is clear that the effect of the asphericity parameter can be neglected, given the geometric variation within the sample. The numerical values used to generate these three geometries are summarized in Table II, where the calculated anterior and posterior radii $R_{a}$ and $R_{p}$ are also included.

\subsection{Constitutive model of the cornea}

Microscopically, the cornea is primarily composed of collagen fibrils that are orientated orthogonally to one another. The directionality of the fibrils changes as they approach the limbus from two sets of orthogonal fibres in the central cornea to a single fibre family circumferentially along the limbal region. In this study, only the orthogonal fibres are modelled. The corneal thickness is 
Table II. Cornea geometric properties for geometries shown in Figure 3.

$\begin{array}{lcccccc}\text { Cornea } & \begin{array}{c}\mathrm{CCT} \\ (\mathrm{mm})\end{array} & \begin{array}{c}\text { PCT } \\ (\mathrm{mm})\end{array} & \begin{array}{c}H \\ (\mathrm{~mm})\end{array} & \begin{array}{c}2 R_{i} \\ (\mathrm{~mm})\end{array} & \begin{array}{c}R_{a} \\ (\mathrm{~mm})\end{array} & \begin{array}{c}R_{p} \\ (\mathrm{~mm})\end{array} \\ \begin{array}{l}\text { Most compliant cornea } \\ \text { Average cornea }\end{array} & 0.514 & 0.737 & 2.095 & 12.400 & 10.222 & 8.721 \\ \text { Stiffest cornea } & 0.572 & 0.779 & 2.324 & 11.252 & 7.972 & 6.745 \\ & 0.630 & 0.821 & 2.553 & 10.104 & 6.275 & 5.206\end{array}$

mainly composed of the stroma, which consists of $80 \%$ water. The cornea is therefore considered to be incompressible as well as viscoelastic. The viscoelastic behaviour can, however, be neglected because all the inflation tests used were performed at the same loading rate of $3.75 \mathrm{mmHg} / \mathrm{min}$.

The role of a constitutive model is to relate the stress in the material to some prescribed level of strain. Because the corneal material experiences relatively large strains, a finite strain formulation is required. One of the most general constitutive models, suitable for finite strains, is the hyperelastic formulation [17]. Hyperelasticity is a constitutive model framework in which the stress-strain relationship is derived from a strain energy density function $\Psi$. Specifically, the Cauchy stress (the stress that is defined in the deformed/loaded configuration) is given by

$$
\sigma={ }_{J}^{1}{ }_{\partial \boldsymbol{E}}^{\partial \Psi} \boldsymbol{F}^{T}
$$

where $\boldsymbol{F}=\left(\begin{array}{c}d \vec{\chi} \\ d \vec{X}\end{array}\right)$ is the deformation gradient, with $\vec{\chi}$ representing the deformed configuration and $\vec{X}$ the undeformed (or reference) configuration. $J$ is the determinant of $\boldsymbol{F}$, and deviation from 1 indicates that the material has undergone a volume change. The Green-Lagrange strain tensor is defined as $\boldsymbol{E}={ }_{2}^{1}\left(\boldsymbol{F}^{T} \boldsymbol{F}-\boldsymbol{I}\right)$, where $\boldsymbol{I}$ is the identity tensor. Hyperelastic formulations are easily implemented in general purpose displacement-based FE codes, because these codes naturally require a constitutive material model that provides the stress, given the strain.

In this study, to account for the orientation of the corneal fibres, an elastic fibre-reinforced model with a neo-Hookean base is chosen (which is available in CalculiX v2.6, an open-source FE solver [18]). This model is similar to the one initially developed by Holzapfel et al. [19] to model arterial walls, and variations of this model have been shown to successfully simulate the corneal structure $[10,20]$. The strain energy density function $(\Psi)$ is composed of a compressible isotropic base material (denoting the ground substance containing no collagen) described using a neo-Hookean law, and an exponential term (denoting the collagen fibre orientations) to form an anisotropic hyperelastic model $[18,21]$ :

$$
\Psi=C_{10}\left(\bar{I}_{1}-3\right)+{ }_{D_{1}}^{1}(J-1)^{2}+\sum_{i=4,6}^{n} \begin{gathered}
k_{1 i} \\
2 k_{2 i}
\end{gathered}\left[e^{k_{2 i}\left\langle\bar{I}_{i}-1\right\rangle^{2}}-1\right],
$$

where $n$ denotes the number of fibre families (only the two orthogonal fibre families are considered in this study), $C_{10}$ and $k_{1 i}$ are stress-like parameters, $k_{2 i}$ is a dimensionless parameter and $D_{1}$ is an incompressibility penalty constant. In this study, a value of $D_{1}=0.4$ was found to be sufficiently small to ensure that only small volume changes occur within the material, while still ensuring numerical stability [22]. $\bar{I}_{1}$ and $\bar{I}_{i}$ denote the invariants of the modified Cauchy-Green deformation tensor $(\overline{\boldsymbol{C}})$. This material model assumes that the fibres are active during tension and inactive during compression [19]. The relevant anisotropic term (cf. Equation (3)) therefore only contributes to the strain energy density function when the fibres are in tension and becomes zero when the fibres are in compression:

$$
\langle x\rangle= \begin{cases}x & \text { if } x \geqslant 0 \text { (tension) } \\ 0 & \text { if } x<0 \text { (compression) }\end{cases}
$$


Although this mathematical description is probably unrealistic (it is unlikely that fibres in compression produce zero resistance), it is a reasonable attempt to model the fact that fibres in tension produce significantly more resistance than fibres in compression. It should also be noted that the isotropic ground substance, which will provide equal resistance in tension and compression, can be used to model that fraction of the fibre resistance that is present both in tension and in compression.

To further simplify the analysis, it is assumed that the fibre stiffness is the same for both sets of fibres [10]. The number of material coefficients is therefore reduced from five to three, as $k_{14}=$ $k_{16}=k_{1}$ and $k_{24}=k_{26}=k_{2}$.

The invariant $\bar{I}_{i}$ is indicative of the stretch measure for the family of collagen fibres and is defined as the square of the stretches in the direction of the directional vector, $\vec{a}_{j}[19,21]$ :

$$
\bar{I}_{i}=\overline{\boldsymbol{C}}: \boldsymbol{A}_{j}, \quad \boldsymbol{A}_{j}=\vec{a}_{j} \otimes \vec{a}_{j}, \quad i=4,6 ; \quad j=1,2,
$$

where $j$ refers to the number of fibre families and $\boldsymbol{A}_{j}$ denotes a structural tensor that characterizes the corneal structure (or material) using the corresponding collagen fibre orientation, also known as a directional vector, $\vec{a}_{j}$. The invariant $\bar{I}_{1}$, associated with the isotropic response, is defined as $[19,21]$ :

$$
\bar{I}_{1}=\operatorname{tr} \overline{\boldsymbol{C}}, \quad \overline{\boldsymbol{C}}=J^{-2 / 3} \boldsymbol{C}, \quad \boldsymbol{C}=\boldsymbol{F}^{T} \boldsymbol{F}
$$

where $\boldsymbol{C}$ is the right Cauchy-Green deformation tensor.

\subsection{Numerical inflation test}

A quarter model of the cornea, using the geometric description outlined in Section 2.2, is generated. The geometric properties reported by Elsheikh et al. [12] are specified for an unloaded cornea, which is equivalent to the undeformed FE geometry. A total of 72620 -noded reduced integration quadratic brick elements are used to mesh the cornea. A local coordinate system is defined for each element, which is used to define the two fibre orientations. Figure 4 illustrates the fibre orientations for a geometry generated using the mean values in Table I. The outer perimeter of the model is clamped, symmetry boundary conditions are applied where required, and a uniform pressure is applied to the posterior surface.

\subsection{Numerical Goldmann applanation tonometry setup}

During GAT, the cornea undergoes biaxial tension (inflation due to the IOP) immediately followed by localized bending (during the applanation process). It is assumed that the cornea-scleral connection has no active role during applanation and the limbal region is therefore completely fixed. Similar to the inflation simulation, the IOP is assumed to be uniformly distributed on the posterior

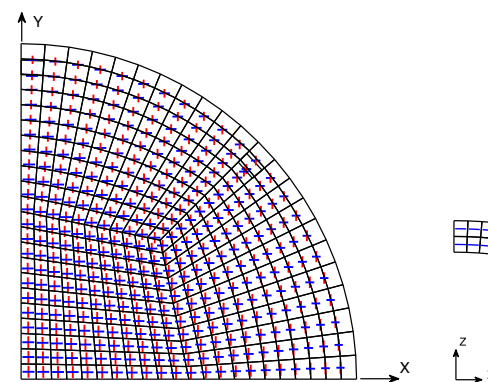

(a)

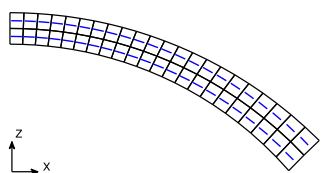

(b)

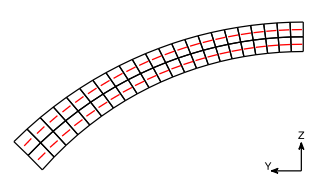

(c)

Figure 4. Preferred fibre orientations as implemented in CalculiX [18], showing (a) the orthogonality of the fibres in the equator-sagittal (XY) plane as well as the fibres along the (b) equator (X) and (c) sagittal (Y) axis following the cornea curvature. 
surface of the cornea, and the applanator is modelled as a rigid body with a prescribed displacement. Similar to the inflation test, only a quarter model of the GAT simulation is required. A total of 4800 20-noded reduced integration quadratic brick elements are used to mesh the cornea. The finer mesh is required to be able to accurately resolve the bending stress through the thickness of the cornea, in the vicinity of applanation. The quarter model of the GAT simulation, using the mean geometric variables in Table I, is shown in Figure 5. Because the FE solver CalculiX cannot resolve contact between quadratic elements, the layer of quadratic elements that are involved in contact is subdivided into linear elements. This step occurs internally in the solver and is only required by the contact algorithm. However, these linear elements do appear in the postprocessing; hence, the apparent mesh refinement is visible in the top layer of the cornea in Figure 5.

The FE output of the GAT simulation can be used to obtain a full ocular response history during the applanation process. The ocular response history is obtained by estimating the point of contact between the applanator and the cornea, and extracting the force and displacement results at this point. The raw data extracted from the FE output are noisy and prevent accurate estimation of the force at full applanation (the force when the applanation radius is $1.53 \mathrm{~mm}$ ). The noisy data are approximated using a simple polynomial $F=c_{1} r+c_{2} r^{2}+c_{3} r^{3}+c_{4} r^{4}$, where $c_{1}, c_{2}, c_{3}$ and $c_{4}$ are estimated using a least squares fit. A typical ocular response history is shown in Figure 6, and it can be seen that the polynomial fit gives a good approximation of the FE data. The ocular response history of the GAT simulation can then be used to estimate the IOP, by dividing the contact force at full applanation by the applanation area. The IOP estimated from a GAT ocular response is denoted IOPG.

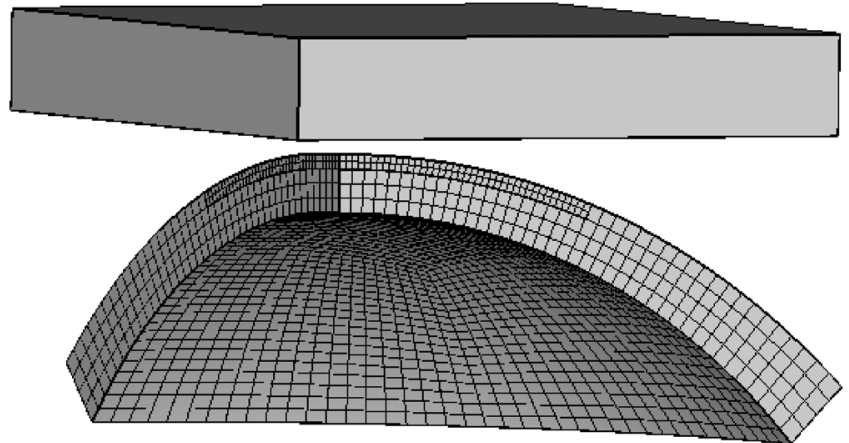

Figure 5. Cornea FE model and rigid applanator used to simulate Goldmann applanation tonometry (GAT).

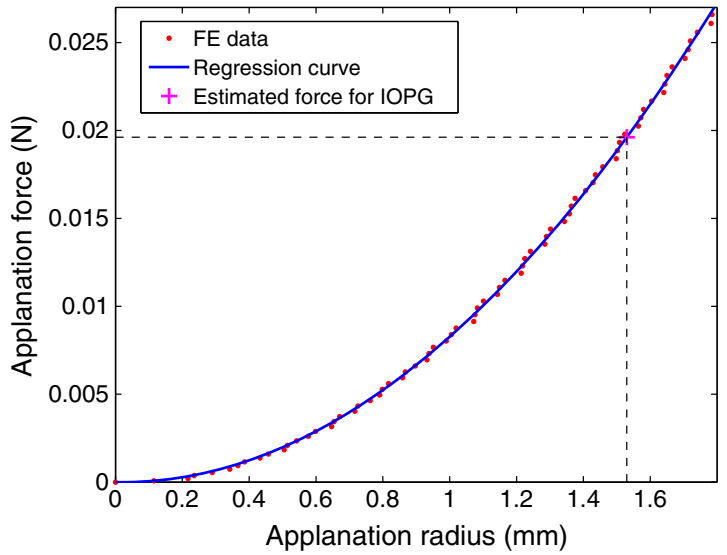

Figure 6. Ocular response history for a GAT simulation assuming the mean values for the geometric variables in Table I, and using a true IOP of $16 \mathrm{mmHg}$. 


\section{NUMERICAL INVESTIGATION}

Now that the development of the FE model is complete, various numerical investigations can be conducted. We will separately investigate the effect on the inflation response of variations in the geometry for a constant set of material parameters, and the effect of varying the material properties while keeping the geometry fixed. This second investigation is the main focus of this paper, because it requires us to undertake the inverse problem of estimating material parameters using experimental data.

\subsection{The effect of geometry}

The first investigation attempts to quantify the variation in inflation response, given the natural variations in the geometries of various corneas. Elsheikh et al. [12] reported the mean and standard deviations of four geometric variables. We now allow each of the four variables to take on either the mean value or the mean value minus one standard deviation or the mean value plus one standard deviation. Therefore, each of the four variables has one of the three possible values. In total, this generates 81 distinct cornea geometries. A numerical inflation test is now conducted for each of these 81 cases.

With the numerical model at our disposal, we can now investigate the effect of varying geometry alone. We keep the material model parameters fixed with values $D=0.4, C_{10}=0.004, k_{1}=$ 0.06839 and $k_{2}=442.7$. These material parameter values produce an inflation curve that almost exactly matches Cornea 5 in Figure 1, if we use the mean values of the geometry reported in Table I (see the reference geometry result in Figure 7). As the geometry varies, we expect some of the simulated inflation responses to be stiffer (less displacement for the same internal pressure) and some of the inflation responses to be more compliant (more displacement for the same internal pressure).

Figure 7 depicts the results of all 81 simulations. Notice that the spread in the observed response, purely due to variations in the geometry, is almost identical to the spread in the experimental data (see Figure 1). One could argue that some of the geometric variations considered are unlikely to ever occur, but this investigation merely attempts to quantify the possible variation in inflation response due to geometry, rather than the likelihood of encountering such a geometry. This study confirms the importance of accurately measuring the geometry of the cornea when attempting to predict the ocular response using simulation tools. Such measurements can be performed routinely.

\subsection{The effect of material parameters}

In the second investigation, we would like to quantify the effect of material property variation on the inflation behaviour. The geometry study was easy to perform, because the variation in geometry

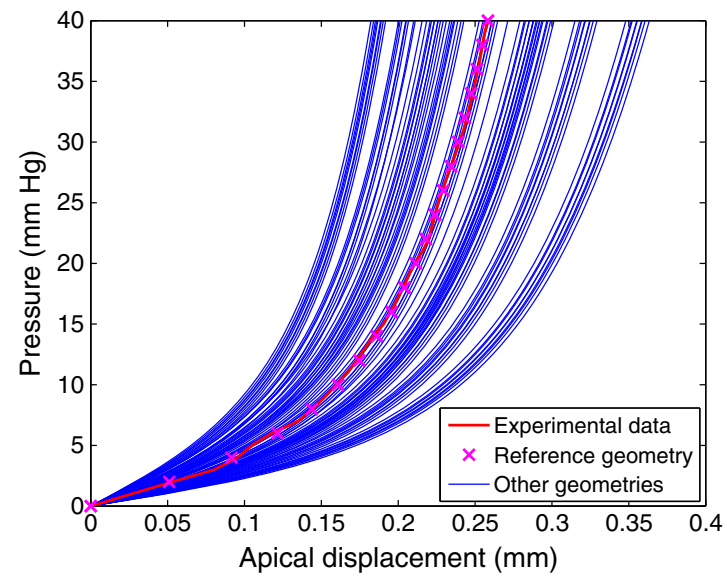

Figure 7. Variation in the inflation response of a cornea due to variations in geometry only. 
is directly measurable. However, there is no consensus in the experimental literature on reference values for material properties. Therefore, a different approach has to be followed. Instead of using known material property variations to predict the variation in inflation response, we will assume that the observed variation in inflation response is due only to material property variation. We, hence, use only the reference geometry and find the material parameters that match each of the experimental inflation curves. In all probability, the true material property variation will be smaller, because there would also be some contribution from geometry variation. Nevertheless, although this approach probably overestimates material property variation, it is preferred to an investigation that simply varies each material parameter by some fixed percentage from an arbitrarily chosen baseline.

The process of finding the material parameters that result in matching the FE results to the experimental curves is described next.

3.2.1. Optimization method used for material parameter identification. The material coefficients required to describe the corneal material are identified by solving an unconstrained optimization problem. The Nelder-Mead simplex method, as implemented in the Matlab optimization routine fminsearch, is used to minimize the root mean square error (RMSE) for the inflation test. The RMSE is composed of the square differences between the apical displacements $(d)$ at given chamber pressures. The objective function is therefore defined as follows:

$$
\min _{\boldsymbol{X}} F(\boldsymbol{X})=\min _{\boldsymbol{X}} \operatorname{RMSE}(\boldsymbol{X})=\sqrt{\frac{\sum_{i=1}^{n}\left(d_{\mathrm{exp}}^{i}-d_{\mathrm{num}}^{i}(\boldsymbol{X})\right)^{2}}{n}},
$$

where $d_{\exp }^{i}$ is the experimental inflation (apical displacement) data set, $d_{\mathrm{num}}^{i}(\boldsymbol{X})$ is the numerical inflation (apical displacement) data set, $n$ is the sample size and $\boldsymbol{X}$ is a vector containing the material coefficients, $C_{10}, k_{1}$ and $k_{2}$ (cf. Equation (3)).

3.2.2. Case 1: Three material coefficients. In the first material parameter identification problem, a distinct material parameter set is found for each of the inflation curves in Figure 1. The optimized material coefficients are given in Table III, with a graphical comparison of the experimental and numerical inflation test data shown in Figure 8(a). It is evident from Figure 8(a) that there is good agreement between the experimental and numerical inflation test data sets. This is further corroborated by the low RMSE values in Table III where the RMSE is less than $0.0047 \mathrm{~mm}$ for each of the curves. The RMSE error can be judged against the typical maximum apical displacement of between 0.18 and $0.35 \mathrm{~mm}$.

Table III. Identified material parameters for Case 1.

\begin{tabular}{lcccc} 
Cornea & $C_{10}(\mathrm{MPa})$ & $k_{1}(\mathrm{MPa})$ & $k_{2}$ & $\mathrm{RMSE}(\mathrm{mm})$ \\
\hline 1 & 0.00291 & 0.0338 & 291.28 & 0.00466 \\
2 & 0.00357 & 0.0219 & 362.33 & 0.00362 \\
3 & 0.04691 & 0.0324 & 1080.51 & 0.00283 \\
4 & 0.03744 & 0.0217 & 1280.07 & 0.00395 \\
5 & 0.00275 & 0.0680 & 435.38 & 0.00176 \\
6 & 0.00906 & 0.1031 & 406.51 & 0.00157 \\
7 & 0.01605 & 0.1602 & 774.71 & 0.00127 \\
8 & 0.00425 & 0.1614 & 667.85 & 0.00143 \\
9 & 0.00328 & 0.1482 & 605.98 & 0.00230 \\
& & & & \\
Mean & 0.01402 & 0.0834 & 656.07 & 0.00260 \\
Standard deviation & 0.01669 & 0.0607 & 338.49 & 0.00123
\end{tabular}




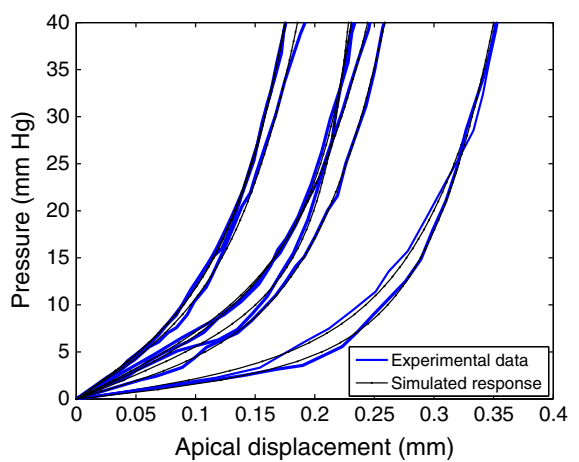

(a)

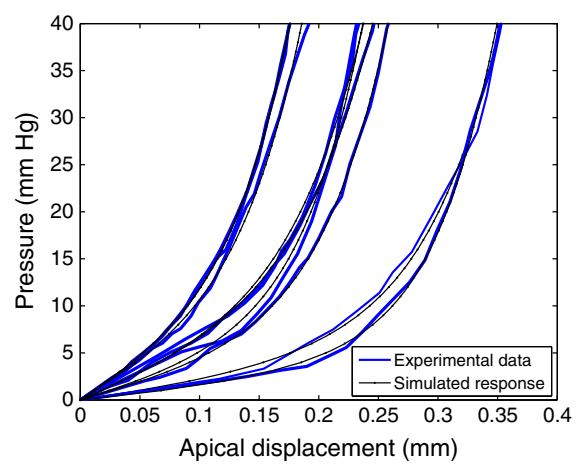

(b)

Figure 8. Inflation response for the calibrated material models for (a) Case 1 and (b) Case 2 (for which $C_{10}$ is fixed to $0.004 \mathrm{MPa}$ ). Experimental data are shown in thick lines, and simulated responses shown in thin lines.

Table IV. Identified material parameters for Case 2, with $C_{10}=0.004 \mathrm{MPa}$.

\begin{tabular}{lccc} 
Cornea & $k_{1}(\mathrm{MPa})$ & $k_{2}$ & RMSE $(\mathrm{mm})$ \\
\hline 1 & 0.0339 & 294.41 & 0.00516 \\
2 & 0.0215 & 368.20 & 0.00365 \\
3 & 0.1054 & 409.55 & 0.00459 \\
4 & 0.0758 & 518.76 & 0.00653 \\
5 & 0.0684 & 442.72 & 0.00187 \\
6 & 0.1049 & 376.44 & 0.00163 \\
7 & 0.1607 & 664.00 & 0.00137 \\
8 & 0.1609 & 667.02 & 0.00143 \\
9 & 0.1496 & 607.08 & 0.00233 \\
& & & \\
Mean & 0.0979 & 483.13 & 0.00317 \\
Standard deviation & 0.0524 & 137.12 & 0.00189
\end{tabular}

Referring to Table III, note that the mean value of $C_{10}$ is 0.01402 , with the standard deviation larger than the estimate itself, at 0.01669 . The Cornea 5 data set leads to the lowest estimate of $C_{10}=0.00275$, while the Cornea 3 data set leads to the highest estimate of $C_{10}=0.04691$. Hence, this first investigation demonstrates that the potential variation in the ground substance stiffness, $C_{10}$, is more than a factor 17 from the lowest to the highest value. The variation in $k_{1}$ is about a factor 7.4 from the highest to the lowest value, while $k_{2}$ varies approximately a factor 4.4 from the highest to the lowest value. This investigation appears to be successful, having established material parameter ranges that can account for the variability in the experimental inflation test responses.

3.2.3. Case 2: Two material coefficients. The next investigation attempts to quantify the effect of suppressing the variation in one of the material parameter values. Specifically, we fix the parameter that had the highest variation in Case $1, C_{10}$. Based on the results from Case 1 , we select the value $C_{10}=0.004 \mathrm{MPa}$. We now repeat all the material parameter identification runs. The results from only calibrating two material coefficients are summarized in Table IV and shown in Figure 8(b). Surprisingly, although we have fixed the parameter which varied by more than an order of magnitude in Case 1, this has had almost no effect on the ability to match the experimental data. The mean RMSE in Table IV increased only marginally from 0.00260 to $0.00317 \mathrm{~mm}$.

Taking into account the experimental noise evident in the data, it would be difficult to argue that the three-parameter fit (Case 1) is indeed superior to the two-parameter fit (Case 2). The obtained material parameter ranges are also affected by fixing $C_{10}$ to $0.004 \mathrm{MPa}$. The $k_{1}$ variable now varies a factor 7.5 from the largest to the smallest value (previously 7.4), while $k_{2}$ now varies a factor 2.3 
Table V. Comparison between material parameters found for Cornea 3.

\begin{tabular}{lccrc} 
Case & $C_{10}(\mathrm{MPa})$ & $k_{1}(\mathrm{MPa})$ & \multicolumn{1}{c}{$k_{2}$} & $\mathrm{RMSE}(\mathrm{mm})$ \\
\hline 1 & 0.04691 & 0.0324 & 1080.51 & 0.00283 \\
2 & 0.00400 & 0.1054 & 409.55 & 0.00459
\end{tabular}

(previously 4.4). Therefore, the material parameter ranges are now reduced compared with the Case 1 result, with a similar ability as before to match the experimental variation. We now have some indication of the required variation in the material parameter values, in order to explain the observed variation in inflation response.

3.2.4. Discussion. We have already considered the variation of the material parameter values within Tables III and IV, but we also have to consider how the material parameter values change from Table III to Table IV. The material properties obtained for a specific cornea (Cornea 3) are compared in Table V. As the value of $C_{10}$ is reduced by a factor $11.7, k_{1}$ increases by a factor 3.3, while $k_{2}$ decreases by a factor 2.6. However, the numerical inflation responses using these two sets of dramatically different material parameter values both approximate the experimental data for Cornea 3 well (RMSE of 0.00283 and $0.00459 \mathrm{~mm}$, respectively). This behaviour is a hallmark of an ill-posed problem. Usually, the ill-posed nature of an inverse problem is demonstrated by adding some noise to the experimental data, and solving the identification problem again. If the change in the solution is significantly more than the level of noise added, the problem is considered ill-posed. In essence, if a problem is ill-posed, then two curves that are nearly identical (only different because of the noise) can be produced by model parameters that are vastly different. In our case, we have two curves (Case 1 and Case 2, respectively) that are nearly identical (both approximate the Cornea 3 experimental data set), but they are produced by material parameter values that are vastly different. Therefore, we can conclude that the identification of material parameter values for a fibre-reinforced hyperelastic material model, using only inflation data, is ill-posed. In essence, the experimental data of the inflation test do not contain sufficient information to uniquely identify the material parameter values.

It is emphasized that the ill-posedness of the inverse problem is not a consequence of making the assumption that the variability in the inflation response is due to material parameter variation alone. We assumed the geometry for Cornea 3, and we calibrated the material model using either two or three free parameters, and we found dramatically different values for these parameters. These different estimates for the material parameters for Cornea 3 are purely due to the ill-posed nature of the inverse problem and cannot be attributed to geometric variation. The variation in material parameters within each of Tables III and IV can, however, be argued to be excessive, because geometric variation alone can explain the different inflation responses, as demonstrated in Section 3.1.

Our attempt to use the inflation experiments to quantify the possible material parameter value variation has to be aborted at this point. The inverse problem is ill-posed, and the proper course of action is to determine the least demanding experimental data that have to be added to the inverse problem, in order to render the problem well-posed. However, some practitioners may be tempted to use the material parameter values obtained to date in further work. They could argue that the agreement between the simulated and experimental responses is good, which gives them confidence that other predictions using these estimated material parameter values may be of use. The next section demonstrates the severe consequences if these unreliable material parameters are used in GAT simulations.

\section{CONSEQUENCE OF ILL-POSEDNESS DEMONSTRATED USING GOLDMANN APPLANATION TONOMETRY}

The effect of the material coefficients is further investigated by comparing the estimated IOP obtained from the ocular response history of a GAT simulation (IOPG). Using all nine cornea 
material data sets, for both Case 1 and Case 2, 18 GAT simulations were conducted to obtain the IOPG. For all the cases, the reference cornea geometry is used (see the mean values in Table I), and an IOP of $16 \mathrm{mmHg}$ is applied. These GAT simulations are now used to demonstrate the effect of varying material properties on IOPG.

The ocular response curves using the material data sets from Case 1 and Case 2 are depicted in Figure 9(a) and (b), respectively. The estimated IOPG values read from these curves are summarized in Table VI. The first result of significance is that for Case 1, the IOPG is strongly correlated to the value for $C_{10}$. The highest $C_{10}$ values are for Corneas $3,4,7$ and 6 , and these cases also produce the highest estimates for IOPG. The results for Case 2 indicate that the GAT simulation is insensitive to variations in $k_{1}$ and $k_{2}$. Recall that the variations in $k_{1}$ and $k_{2}$ were factors 7.5 and 2.3 , respectively, but the estimated IOPG values only vary between 19.66 and $20.54 \mathrm{mmHg}$.

To date, it has been established that the numerical GAT simulation is sensitive to $C_{10}$ and insensitive to $k_{1}$ and $k_{2}$. The other consistent numerical observation that can be made from the GAT simulations is that the cornea stiffness does contribute to the IOPG. The magnitude of the cornea stiffness contribution to the IOPG is at least $2.54 \mathrm{mmHg}$ for Case 1 (Cornea 5), while for Case 2, this contribution varies between 3.66 and $4.54 \mathrm{mmHg}$.

These GAT simulations, using calibrated material coefficients, demonstrate the main result of the study. It would be entirely plausible that a group of researchers calibrate three material coefficients to match the experimental inflation data. It would be equally reasonable that another group of researchers starts off by assuming a constant stiffness for the cornea ground substance, ascribing all differences in cornea stiffness to the fibres. This second group would only calibrate two material coefficients in order to match the same experimental inflation data. However, these two groups

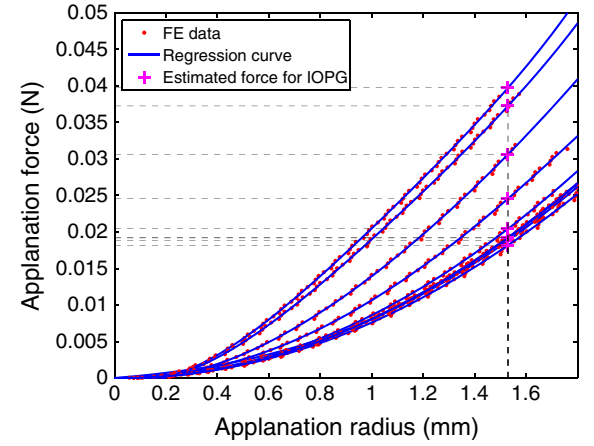

(a)

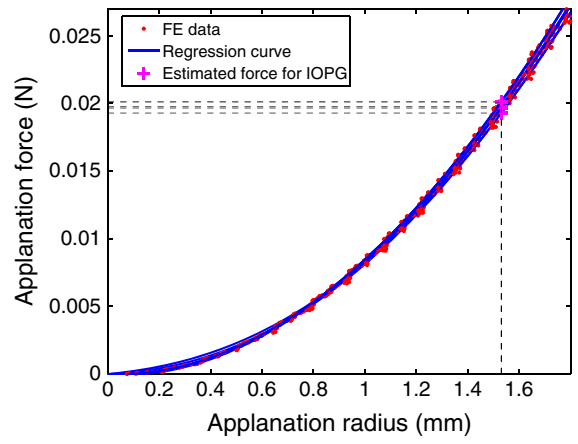

(b)

Figure 9. Ocular response history during the GAT simulation, using the material parameters for (a) Case 1 and (b) Case 2 (for which $C_{10}$ is fixed to $0.004 \mathrm{MPa}$ ).

Table VI. Summary of the IOPG derived from the ocular response history at the point of full applanation for the reference cornea geometry, and a true IOP of $16 \mathrm{mmHg}$ ).

\begin{tabular}{lcc} 
Cornea & $\begin{array}{c}\text { IOPG }(\mathrm{mmHg}) \\
\text { Case } 1\end{array}$ & $\begin{array}{c}\text { IOPG }(\mathrm{mmHg}) \\
\text { Case 2 }\end{array}$ \\
\hline 1 & 19.66 & 19.66 \\
2 & 19.20 & 19.69 \\
3 & 40.53 & 20.14 \\
4 & 37.99 & 20.16 \\
5 & 18.54 & 20.04 \\
6 & 25.05 & 20.10 \\
7 & 31.24 & 20.54 \\
8 & 20.85 & 20.54 \\
9 & 19.56 & 20.48
\end{tabular}


of researchers would report very differently on the effect that varying material properties has on estimated IOPG. The first group would claim that GAT is very sensitive to material parameters. In contrast, the second group would claim that GAT is not sensitive to material parameters, which agrees with the premise of Goldmann and Schmidt [5] on which GAT is based.

\section{ALTERNATIVE LOADING STATES}

It is clear that two very different conclusions can follow from the results regarding the sensitivity of GAT with regard to material properties. In this section, it is demonstrated that the ambiguity regarding the effect of material property variation is a consequence of the interplay between the chosen material model and the data used to calibrate this model. We conduct a simulation of a simply loaded corneal material, using the material model parameters calibrated against the inflation test data. In the simulation, uniaxial tension is applied, followed by a bending load. The uniaxial tension case produces loading that is similar to that experienced during inflation, while the subsequent bending load produces a loaded state that is similar to the localized bending experienced during applanation (local flattening of the cornea due to contact with an applanator).

An FE model of a thin strip is developed, depicted in Figure 10(a), with a length of $5 \mathrm{~mm}$, a width of $1 \mathrm{~mm}$ and a height (or thickness) of $0.5 \mathrm{~mm}$. The elastic fibre-reinforced material model is implemented with fibres orientated in the $0^{\circ}$ and $90^{\circ}$ orientations as shown in Figure 10(b). The two load conditions are depicted in Figure 10(c) and (d), respectively. The strip is completely fixed at the one end, and the respective loads are applied at the opposite end. A mesh size of $20 \times 4 \times 8$ is employed for both loading scenarios, and reduced integration 20-noded quadratic brick elements are used. The tensile load, applied as a negative pressure, is applied linearly over time $t \in[0 ; 0.5]$, whereafter it remains constant at $-0.01 \mathrm{MPa}$. The bending load, applied as a pressure on four elements, is zero up to $t=0.5$, whereafter it increases linearly up to $0.0014 \mathrm{MPa}$ at $t=1$. The strip simulation is performed for Cornea 3, using the material parameter values from Case 1 and Case 2.

The results from the strip simulations are illustrated in Figure 11. When comparing the results for both calibration cases (i.e., calibrating using three and two material coefficients), it is evident that the axial displacements are in close agreement during the application of only the axial load.

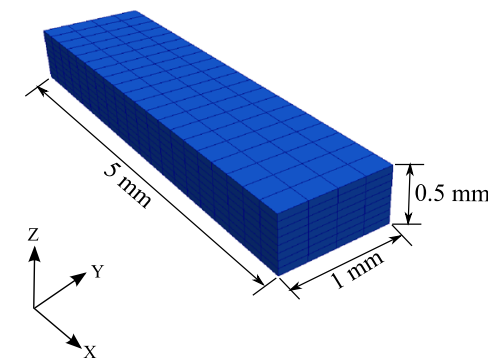

(a)

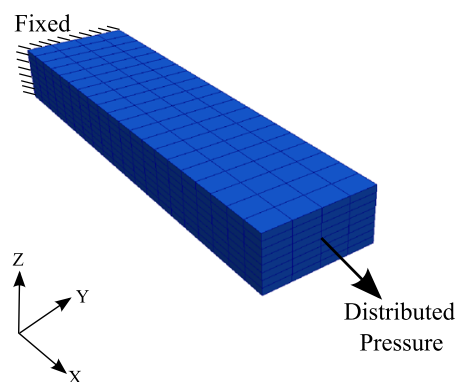

(c)

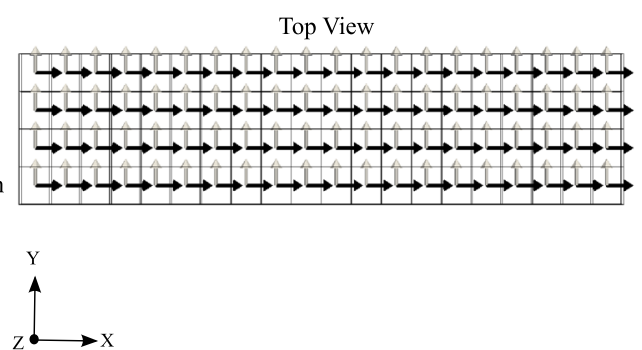

(b)

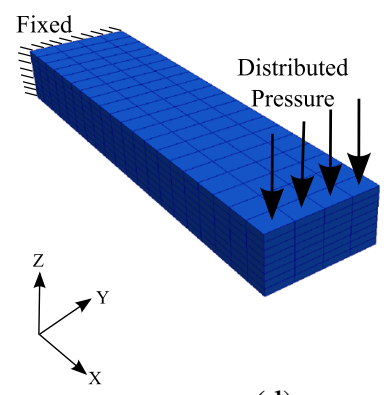

(d)

Figure 10. FE model of the thin strip illustrating (a) the strip dimensions, (b) the $0^{\circ}$ and $90^{\circ}$ fibre orientations, with the prescribed boundary conditions for the (c) tension and (d) bending loading scenarios. 


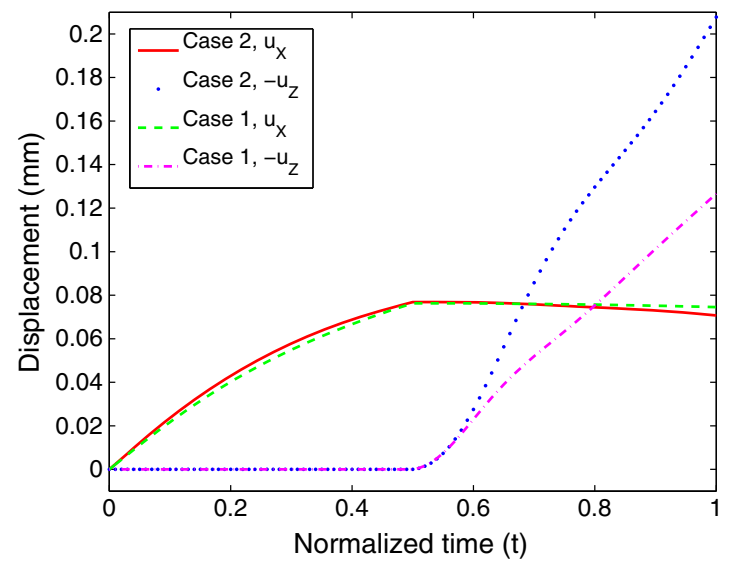

Figure 11. Results from the strip simulations for a tension followed by bending loading scenario. Displacements in the axial $(x)$ and transverse $(y)$ directions are plotted against normalized time. Axial loading increases during the interval $t \in[0.0 ; 0.5]$, and bending load increases while axial load is held constant during the interval $t \in[0.5 ; 1.0]$.

This result is similar to that of the inflation test where the experimental and numerical inflation test data were in good agreement for both instances of calibrated material coefficients. Considering the form of the strain energy density function (cf. Equation (3)), both the ground substance and the fibres are loaded during tension. Both these terms also contribute during inflation. Hence, it should be anticipated that if the inflation response of the two material sets is similar, then so will be the uniaxial tension response. The response of the two materials during the subsequent application of the bending load, however, is distinctly different. The transverse response of the Case 1 cornea is substantially stiffer than that of the Case 2 cornea, a consequence of the dramatic difference between the $C_{10}$ parameters in each case. Because the material properties were calibrated using only inflation data, the contribution of each component (ground substance and fibres) could not be determined uniquely. Under the application of a bending load, such as experienced during GAT and in this strip simulation, the ground substance dominates the response, and the weakness of a calibration method based on matching only inflation data is exposed.

This simple strip simulation clearly demonstrates why inflation data are insufficient to calibrate our specific choice of material model. The inflation test produces loading that does not differentiate between the ground substance and the fibres. Hence, as long as the effective stiffness of the material is correct, the correct inflation response will be obtained. However, the relative contribution of the ground substance and fibres could be incorrect. A bending load case, on the other hand, has the ability to differentiate between the ground substance and the fibres. Which of the two material model assumptions (i.e. calibrating two or three material coefficients) is more accurate cannot be determined at this point. Indeed, both could be wrong. The point is, however, that it is a dangerous practice to calibrate a material model using a specific load state and then to use this calibrated material model in a load state that is substantially different. This is especially important for biomaterials, which often have a complicated microstructure and may behave dramatically different as the load state varies. Although these words of caution seem obvious, it is still a common practice in numerical simulations of corneal biomechanics to only calibrate using inflation data and then to predict the corneal biomechanics for other scenarios.

\section{CONCLUSIONS}

The effect of varying corneal material properties on GAT has been of interest for some time. One seemingly attractive approach is to answer this question using numerical simulation making use of the FE method. However, a calibrated material model is required before these simulations can be performed. The actual calibration process requires the solution of an inverse problem. The material 
coefficients are altered using some optimization routine, in order to minimize the discrepancy between simulated and experimental data. The data used most often to calibrate corneal material models are obtained from inflation experiments.

At face value, it would seem reasonable to accept a calibrated material model, if there is good agreement between the experimental and simulated data. However, we have demonstrated in this paper that this approach could have serious limitations. In this paper, we selected an anisotropic hyperelastic model consisting of an isotropic ground substance reinforced with nonlinear elastic fibres. We started off by calibrating three material coefficients, followed by a case where only two material parameters were calibrated. Both cases provided good agreement between simulated and experimental inflation data. However, once these calibrated material models were used to predict the response of the cornea during applanation, dramatically different results were obtained. It was then demonstrated that the reason for this behaviour is the inability of inflation experiments to differentiate between stiffness contributions either from the ground substance or from embedded fibres. Calibrating our selected corneal material model using only inflation data is an ill-posed problem. This ill-posedness can be addressed by including additional experimental data during the calibration process. It is suggested that when intended to model a bending loading state, for instance, the additional experimental data describing the corneal behaviour during bending should be employed during the calibration process. Experimentalists are therefore urged to conduct additional experimental studies in which the corneal behaviour under various loading states is investigated.

Although this work was focussed on corneal material model calibration using only inflation data, the lessons learned have wider application. Biomaterials are often complex with highly nonlinear behaviour. When calibrating material models designed to describe these biomaterials, take caution to include the load states that are expected to be present in the final application of the models. If these load states are omitted during the calibration process, it becomes possible (even likely) that completely misleading predictions will be made regarding the behaviour of the material.

\section{ACKNOWLEDGEMENTS}

This study was funded by the Advanced Mathematical Modelling competency area within Modelling and Digital Science at the Council for Scientific and Industrial Research in South Africa.

\section{REFERENCES}

1. Bryant MR, McDonnell PJ. Constitutive laws for biomechanical modeling of refractive surgery. Journal of Biomechanical Engineering 1996; 118(4):473-481. DOI: 10.1115/1.2796033.

2. Liu J, Roberts CJ. Influence of corneal biomechanical properties on intraocular pressure measurement: Quantitative analysis. Journal of Cataract \& Refractive Surgery 2005; 31(1):146-155. DOI: 10.1016/j.jcrs.2004.09.031.

3. Kwon T, Ghaboussi J, Pecknold D, Hashash Y. Effect of cornea material stiffness on measured intraocular pressure. Journal of Biomechanics 2008; 41(8):1707-1713. DOI: 10.1016/j.jbiomech.2008.03.004.

4. Kniestedt C, Punjabi O, Lin S, Stamper RL. Tonometry through the ages. Survey of Ophthalmology 2008; 53(6): 568-591. DOI: 10.1016/j.survophthal.2008.08.024.

5. Goldmann H, Schmidt T. Über Applanationstonometrie (applanation tonometry). Ophthalmologica 1957; 134: 221-242. in Ritch R, Caronia RM. Classic Papers in Glaucoma, Kugler Publications 2000; 155-162.

6. Kabanikhin SI. Definitions and examples of inverse and ill-posed problems. Journal of Inverse and Ill-Posed Problems 2008; 16:317-357. DOI: 10.1515/JIIP.2008.069.

7. Studer H, Riedwyl H, Büchler P. Importance of multiple loading scenarios for the identification of material coefficients of the human cornea. Computer Methods in Biomechanics and Biomedical Engineering 2012; 15(1): 93-99. DOI: 10.1080/10255842.2011.552184.

8. Elsheikh A, Anderson K. Comparative study of corneal strip extensometry and inflation tests. Journal of The Royal Society Interface 2005; 2(3):177-185. DOI: 10.1098/rsif.2005.0034.

9. Elsheikh A, Wang D, Kotecha A, Brown M, Garway-Heath D. Evaluation of Goldmann applanation tonometry using a nonlinear finite element ocular model. Annals of Biomedical Engineering 2006; 34(10):1628-1640. DOI: 10.1007/s10439-006-9191-8.

10. Pandolfi A, Manganiello F. A model for the human cornea: constitutive formulation and numerical analysis. Biomechanics and Modeling in Mechanobiology 2006; 5(4):237-246. DOI: 10.1007/s10237-005-0014-x.

11. Studer H, Larrea X, Riedwyl H, Büchler P. Biomechanical model of human cornea based on stromal microstructure. Journal of Biomechanics 2010; 43(5):836-842. DOI: 10.1016/j.jbiomech.2009.11.021. 
12. Elsheikh A, Wang D, Pye D. Determination of the modulus of elasticity of the human cornea. Journal of Refractive Surgery 2007; 23(8):808-818.

13. Okrasinski W, Plociniczak L. Bessel function model of corneal topography. Applied Mathematics and Computation 2013; 223:436-443.

14. Plociniczak L, Okrasinski W. Regularization of an ill-posed problem in corneal topography. Inverse Problems in Science and Engineering 2013; 21(6):1090-1097.

15. Carney LG, Mainstone JC, Henderson BA. Corneal topography and myopia. A cross-sectional study. Investigative Ophthalmology \& Visual Science 1997; 38(2):311-320.

16. Liou HL, Brennan NA. Anatomically accurate, finite model eye for optical modeling. Journal of the Optical Society of America A 1997; 14(8):1684-1695.

17. Holzapfel GA. Nonlinear Solid Mechanics: A Continuum Approach for Engineering (1st edn). John Wiley \& Sons: New York, USA, 2000.

18. Dhondt GDC. CalculiX: A free software three-dimensional structural finite element program, 2011.

19. Holzapfel GA, Gasser TC, Ogden RAYW. A new constitutive framework for arterial wall mechanics and a comparative study of material models. Journal of Elasticity 2000; 61:1-48. DOI: 10.1023/A:1010835316564.

20. Pandolfi A, Holzapfel GA. Three-dimensional modeling and computational analysis of the human cornea considering distributed collagen fibril orientations. Journal of Biomechanical Engineering 2008; 130(6):061006-1-061006-12. DOI: $10.1115 / 1.2982251$.

21. Dhondt GDC. The Finite Element Method for Three-dimensional Thermomechanical Applications. John Wiley and Sons Ltd: The Atrium, Southern Gate, Chichester, West Sussex PO19 8SQ, England, 2004.

22. Botha N. Effect of numerical modelling assumptions on the simulated corneal response during Goldmann applanation tonometry. Masters Dissertation, University of Pretoria, South Africa, 2014. 